\title{
REFORMAS EDUCATIVAS EN CHILE: UNA MIRADA DESDE EL ENFOQUE DE GÉNERO
}

\author{
Raquel del Carmen Flores Bernal ${ }^{1}$ \\ Emivaldo Silva Nogueira ${ }^{2}$ \\ Viviana Rebufel ÁlvareZ ${ }^{3}$
}

\begin{abstract}
RESUMEN: El presente estudio tiene como objetivo analizar las reformas del sistema educativo en Chile entre los años 1965 bajo el gobierno de Eduardo Frei Montalva; 1973, con Salvador Allende Gossens; y 1981, con la privatización y la municipalización de la educación durante el régimen militar de Augusto Pinochet Ugarte. Estos tres momentos políticos hicieron cambios importantes en la educación en Chile, expandiendo y estableciendo parámetros educativos que cruzaron esos momentos históricos y han llegado a nuestros días. Sin embargo, dentro de este tema, la cuestión de género fue poco discutido. Por lo tanto, en este estudio, analizaremos cómo la educación, en estos tres períodos históricos, enfrentó el tema del género y las desigualdades educativas existentes entre hombres y mujeres.
\end{abstract}

Palabras-clave: Reformas. Educación. Chile. Género.

\section{EDUCATIONAL REFORMS IN CHILE: A LOOK FROM THE GENDER PERSPECTIVE}

\begin{abstract}
This study aims to analyze the reforms of the educational system in Chile between 1965, under the government of Eduardo Frei Montalva; 1973, with Salvador Allende Gossens; and 1981, with the educational privatization and municipalization during the military regime of Augusto Pinochet Ugarte. These three political moments made important changes in education in Chile, expanding and establishing educational parameters that crossed those historical moments and have reached our days. However, within this issue, the gender issue was little discussed. Therefore, in this study, we will analyze how education, in these three historical periods, faced the issue of gender and educational inequalities between men and women.
\end{abstract}

Keywords: Reforms. Education. Chile. Gender.

\footnotetext{
La investigación fue financiada por la Universidad Bernardo O’Higgins, Chile

1.Universidad Bernardo O’Higgins - Santiago de Chile, Chile. E-mail: raquel.flores@ubo.cl

2.Universidad Bernardo O’Higgins - Santiago de Chile, Chile. E-mail: filliusorion@hotmail.com

3.Universidad de Valencia - Santiago de Chile, Chile Email: vipazre@alumni.uv.es

Editor de Sección: Rodrigo Cornejo
} 


\title{
REFORMAS EDUCACIONAIS NO CHILE: UM OLHAR A PARTIR DA PERSPECTIVA DE GÊNERO
}

\begin{abstract}
RESUMO: O atual estudo objetiva analisar as reformas do sistema educacional no Chile entre os anos 1965, sob o governo de Eduardo Frei Montalva; 1973, com Salvador Allende Gossens; e 1981, com a privatização e a municipalização da educação durante o regime militar de Augusto Pinochet Ugarte. Esses três momentos políticos realizaram mudanças importantes no fazer educação no Chile, ampliando e estabelecendo parâmetros educacionais que transpassaram tais momentos históricos, e chegaram até nossos dias. Todavia, dentro desta temática, a questão de gênero pouco foi debatida. Portanto, neste estudo, faremos uma análise de como a educação, nestes três períodos históricos, enfrentou a questão de gênero e as desigualdades educacionais existentes entre homens e mulheres.
\end{abstract}

Palavras-chave: Reformas. Educação. Chile. Gênero.

\section{Introducción}

$\mathrm{D}$ urante los últimos cincuenta y cinco años nuestro país ha vivido profundas transformaciones políticas, económicas, sociales y culturales. En este contexto la educación formal destaca como uno de los ámbitos donde se han desarrollado mayores cambios, registrándose diversas propuestas de Reformas al Sistema Educacional, que distintos gobiernos han desarrollado o intentado implementar. En 1965 el gobierno del demócrata cristiano Eduardo Frei Montalva (1964-1970) promovió una de las reformas que a juicio de algunos autores ha sido la más ambiciosa y cuyo objetivo principal fue acelerar la ampliación de la cobertura escolar.

La Escuela Nacional Unificada (ENU) fue uno de los principales emblemas del gobierno de Salvador Allende Gossens durante el gobierno de la Unidad Popular (1970-1973). El proyecto planteaba una reforma profunda al sistema educativo chileno, con el objetivo de estructurar la educación de acuerdo a criterios igualitarios y equitativos. A comienzos de los años 80 el gobierno militar (1973-1990) llevó a cabo una de las reformas estructurales más grandes, transformando profundamente el modelo de financiamiento y gestión del sistema escolar.

Este artículo hace una breve descripción de cada una de estas tres propuestas de reforma educativa para luego centrarse en un análisis de éstas desde el enfoque de género. Como es sabido, cada sociedad y cada cultura tiene y reproduce una construcción social de género, una idea compartida de lo que es ser hombre y ser mujer en ese lugar y momento histórico y esta concepción está implícita en todos los ámbitos sociales, también en los sistemas educativos y por cierto en las propuestas de reforma.

\section{Reforma Educativa de 1965: Gobierno de Eduardo Frei Montalva}

Durante la década del 1960 en el continente americano se vivían tiempos de cambio y Chile no era la excepción. A fines de 1964, asumió el gobierno el presidente Eduardo Frei Montalva; durante el año 1965 se inició la planificación de una reforma profunda y gradual de la educación chilena. En diciembre del mismo año se firmó el decreto que dio inicio formal al proceso de reforma. 
Para el gobierno de Frei Montalva la educación era el eje de una estrategia general de cambio social que se expresaba como "Revolución en Libertad", puesto que se consideraba esencial la formación de los recursos humanos que requieren el crecimiento y modernización de la economía, así como la integración sociopolítica de las mayorías excluidas. También lo era para mantener un orden social sobre bases de consenso y cooperación (ROSSETTI, 1988). La reforma iniciada en 1965 se abocó a la reestructuración de todo el sistema educacional y llevó a cabo tres tareas: ampliación e igualación de las oportunidades educativas; cambios educacionales en función del desarrollo económico, social y político; e implementación de un nuevo modelo pedagógico que modificó los programas y las relaciones sociales dentro de la escuela. En corroboración, el exministro Sergio Molina señala:

El propósito principal de la Reforma iniciada a partir de 1965 fue reformular los objetivos y los medios para que el sistema educacional pudiera responder a los requerimientos del desarrollo del país y a los avances científicos y tecnológicos de esos tiempos (Molina, 2004, p.7).

Además de presentar el propósito básico de la reforma educativa, Molina agrega que esta fue una reforma que:

[...] se fundaba en la convicción de que la educación era un sector desde el cual el Estado podía contribuir a superar las injusticias sociales, aportando, a través de la formación de los alumnos, tanto a fortalecer el crecimiento económico como a garantizar el orden democrático (Molina, 2004, p. 9).

En la idea de mejorar el nivel educacional de los sectores menos favorecidos, se incrementó el nivel básico de enseñanza, de seis a ocho años, cambiando la denominación de primario por básico; se acortó la secundaria a cuatro años, y se definió que sus dos ramas (humanística y profesional) permitan el acceso a la Universidad, porque disminuyen las diferencias de los programas de estudios de ambas orientaciones.

En sus aspectos propiamente pedagógicos, se modificaron los planes y programas de enseñanza (básica y media) de "acuerdo a una concepción curricular inspirada en las propuestas de Benjamín Bloom y Ralph Tyler. Se reformaron también, bajo la misma inspiración, los programas de las diversas especialidades de la educación media técnico-profesional” (NUÑEZ, 1990, p. 123). Eduardo Frei Montalva, en una carta al Congreso Nacional en 1968, declaró que el resultado de esta reforma se hizo notable en el aumento vertiginoso de la matrícula en la educación básica en las escuelas:

El incremento ha sido de más de 500.000 nuevas matrículas entre 1965 y 1967, o sea, un 26\% en tres años, que supera varias veces cualquier incremento habido en un período similar en la historia de Chile. En 1968 el 93\% de los niños en edad escolar está asistiendo a clases, es decir, una de las tasas más altas del mundo (FREI, 1968, p. 38).

En el primer ciclo de la educación básica, los programas se organizaron en unidades programáticas. Con ellas se intentaba relacionar un tema central con las necesidades e intereses de los niños y con las características del medio en el que se desenvuelven. Las unidades programáticas tales como "La comunidad en que vivimos", "El paisaje al servicio del hombre", "Una herramienta para ser felices, el trabajo" y otras se centraban en el desarrollo de habilidades, la incorporación de experiencias de niños y niñas, y la adquisición de nuevos conocimientos. 
En el segundo ciclo de la enseñanza básica se buscaba establecer conocimientos afines entre sí, que constituyeran áreas programáticas y asignaturas, diversificando las experiencias de niños y niñas sin atomizarlas y facilitando un desarrollo analítico, reflexivo y crítico. En la educación media los objetivos educacionales se centraron en el desarrollo de la personalidad, la participación en el cambio social y la vida del trabajo. Se eliminaron los exámenes entre el nivel primario y secundario y se redujo la nota de promoción en el octavo año básico (de 4 a 3,5). La educación normal ascendió del nivel secundario al superior.

Desde el punto de vista de la transmisión pedagógica, la reforma introdujo cambios importantes en la dimensión instructiva y en la dimensión valórica o regulativa de la educación. Para apoyar la reforma en el año 1967 se fundó el Centro de Perfeccionamiento, Experimentación e Investigaciones Pedagógicas, organismo que promovería el perfeccionamiento masivo de profesores y profesoras en servicio, a fin de capacitarlos para participar activamente en los cambios implantados (NUÑEZ, 1990). En abril de 1970 se creó la Junta Nacional de Jardines Infantiles lo que dio gran impulso a la educación pre básica.

\section{La Escuela Nacional Unificada: Propuesta de Reforma Educativa en el Gobierno de Salvador Allende Gossens}

El gobierno del presidente Salvador Allende puso en marcha una estrategia educacional de doble dimensión. Por una parte, desarrolló una política de “democratización”2 que, junto con profundizar la modernización y expansión cuantitativa iniciada por su antecesor, amplió notablemente las posibilidades de participación de las fuerzas sociales en la discusión del rumbo que tomaría la educación dentro del proceso de cambios iniciado en Chile en 1970. Por otra parte, elaboró, propuso y defendió un proyecto de reforma profunda de la enseñanza regular que recogía una alternativa concepción ideológica y educacional (NUÑEZ, 1990).

En la propuesta de Escuela Nacional Unificada (ENU) ${ }^{3}$ desarrollada por el gobierno de la Unidad Popular, presidido por Salvador Allende Gossens (1970-1973), se observa una marcada continuidad con la política educacional del gobierno anterior. En la práctica sólo se acentúa la dimensión de distribución social de la educación sin que se llegue a modificar el sistema. El gobierno realiza fuertes inversiones para profundizar la política de igualdad de oportunidades del periodo anterior (ROSSETTI, 1988).

Durante esta administración la atención en el ámbito educacional está centrada en aumentar la cantidad, calidad y diversidad de los programas asistenciales y las condiciones materiales de los establecimientos. Es así como se observa un aumento significativo en el número de textos escolares entregados a cada establecimiento educacional y en el equipamiento que se entrega a la enseñanza técnico-profesional. El perfeccionamiento docente se profundiza y masifica a través de políticas estatales y la educación popular de adultos adquiere una relevancia que hasta ahora no se había dado.

El gobierno del presidente Allende, en sus dos primeros años, dedicó sus mejores esfuerzos a llevar a sus más altos niveles la expansión de la cobertura del sistema y a democratizar la oferta educativa y la gestión del sistema, sin intentar una nueva reforma educacional (NUÑEZ, 1990, p. 138).

En el 1973, se establecen las bases de la reforma conocida como Escuela Nacional Unificada -ENU. En los aspectos propiamente pedagógicos, se introducen nuevos criterios pedagógicos, que proponen una educación común obligatoria de 12 años para todos los niños y niñas y se establece la enseñanza parvularia obligatoria. 
El elemento central del proyecto era el vínculo de la educación con el trabajo fabril, siendo este último parte importante de los contenidos del currículo. Esta propuesta reformista generó un fuerte conflicto político y no pudo ser legislada, ya que el gobierno lo retiró de la discusión en el Congreso por la gran resistencia que generó. En la práctica, la política educacional del gobierno de Allende fue la continuación de la anterior reforma educacional, ampliando la cobertura del sistema.

\section{Reforma Educacional de 1981: Privatización y Municipalización ${ }^{4}$ de la Educación durante el Régimen Militar (1973-1989)}

Durante el Gobierno Militar, presidido por Augusto Pinochet Ugarte desde 1973 a 1989, se realiza

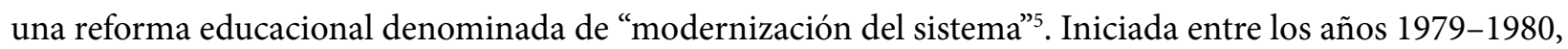
esta reforma puso el acento en la reestructuración administrativa del sistema. El que hacer pedagógico debía inspirarse en los principios del humanismo cristiano nacionalista que había adoptado la Junta Militar de Gobierno. Se relegitimaron aspectos de la educación tradicional como la disciplina, la autoridad docente y del texto, altas exigencias de rendimiento, exámenes, etc. En este periodo la educación se somete a las leyes del mercado y se la presenta como un servicio, amarrada a la oferta y la demanda. El papel regulador del estado se debilita, generándose un esquema que invita a la libre competencia de los sectores público y privado.

Los cambios en el currículo se orientan hacia la eficiencia administrativa y económica de la educación. Y a excluir todos los contenidos relacionados con la reflexión crítica respecto a la formación ciudadana, la participación social y formación en derechos humanos. En términos administrativos los supuestos básicos de esta reforma, es que se asocia la calidad de la educación a la descentralización del sistema, al traspaso de las mismas a la gestión privada y a la libertad de elección de los establecimientos, en función de la calidad de los servicios educativos. Con esta medida se busca disminuir el peso de la gestión estatal e incentivar la participación local y privada.

Entre los años 1973 y 1989, hubo trece decretos del Ministerio de Educación que modifican los planes y programas de estudio. Sin embargo, sólo dos momentos de intervención son de consecuencias importantes sobre el sistema: en 1974, con el Ministerio de Educación bajo el control de la Armada, y en 1980-1981, bajo el ministro Alfredo Prieto, en que se decretan los planes y programas de estudio de la educación básica y media. Las medidas de 1974 son de ideologización del currículo, las de 1980-1981 se inscriben en un contexto de políticas descentralizadoras y apuntan a su flexibilización (COX, 1997, p. 56).

Las modificaciones de 1974 responden al contexto político, expresándose especialmente en los programas de Historia y Geografía y Ciencias Sociales de la Enseñanza Media (COX, 1997).

Los cambios corresponden a la exclusión de ciertos tópicos y la introducción de algunos nuevos, acordes con la visión militar y de la derecha tradicional, que articulan un nuevo concepto de nación. Además, se eliminan temas referidos a América Latina, como la integración social, la sociedad pluralista, el subdesarrollo y la dependencia externa, se celebra a España como referencia relevante, con una historia patria resultante de héroes y personalidades y una visión de la economía desconectada de la historia.

En marzo de 1990, en los últimos días del régimen del general Pinochet se aprobó la Ley Orgánica Constitucional de Enseñanza n. 18.962 -LOCE. El currículo vigente hasta el año 1999, fue fijado en mayo de 1980, para la Educación Básica y diciembre de 1981 para la Media, fundamentado en la ideología neoliberal. 
El currículo revela que carecían de consensos mínimos sobre sus orientaciones y valores, representando sólo a un parte de la sociedad por lo que carecía de legitimidad. Esta parcialidad afectaría la riqueza cultural que la escuela transmitirá durante dicho periodo. De hecho, la mirada estaría más concentrada en el problema del control de la transmisión escolar que en sus contenidos (COX, 1997).

Los nuevos programas son más breves y generales. El currículo de 1981 está construido a partir de objetivos de "mediana especificidad", en ellos se explicitan menos contenidos, a la vez que se eliminan dos categorías que tenían los programas de la reforma del 65, estas son las "actividades" y "sugerencias metodológicas" que se realizan al profesorado. Se plantean objetivos programáticos mínimos tanto para la educación básica como para la enseñanza media. El criterio fundante del diseño del nuevo currículo es la flexibilidad.

La dirección fundamental de las innovaciones implementadas bajo el alero de esta nueva reforma, tienen más que ver con el intento de traspasar decisiones curriculares y pedagógicas del aparato central del Ministerio de Educación a los establecimientos educacionales y profesorado, que con los contenidos de los programas (COX, 1997). En la enseñanza básica, los planes de estudio fueron abiertos a las decisiones de los directores de establecimientos, los que "fueron autorizados a distribuir el tiempo de trabajo semanal entre los distintos ramos de acuerdo a la dotación de recursos de la escuela ('flexibilidad' en este caso significa la posibilidad de dejar de impartir un ramo determinado y reasignar horas)" (COX, 1997, p. 78).

La enseñanza media científico-humanista, queda constituida por dos ciclos de dos años cada uno: el primero y segundo años son de formación general dada por asignaturas comunes; en el tercero y cuarto año hay un plan común y uno electivo. Excluyéndose del plan común las asignaturas de Física y Química, quedando sólo Biología de la tríada tradicional de las ciencias naturales, suprimiéndose además un segundo idioma extranjero. En el plan electivo cada establecimiento según sus posibilidades determina los ramos que ofrecerá (ubicación regional, demandas de la comunidad, entre otros criterios).

\section{Una Mirada desde el Enfoque de Género ${ }^{6}$}

Es a partir de la reforma educacional de 1965 (Gobierno de Frei Montalva) que se presenta una gran expansión de la educación mixta y de la incorporación de las mujeres a todos los niveles de enseñanza. Las escuelas secundarias industriales y agrícolas se abren a las mujeres, implantando la coeducación en dichos establecimientos (ROSSETTI, 1988). La concepción de hombre y mujer en los planes y programas de 1965, se encuentra materializada en las observaciones específicas en la educación técnico manual y la educación básica. En la fundamentación de los programas de estudio del área tecnológica se establece que:

Los cambios sociales y económicos por los que atraviesa nuestro país afectan en forma notoria al núcleo básico de la sociedad, la familia. En primer lugar, la incorporación de la mujer a la vida del trabajo, no solamente del trabajo doméstico sino del trabajo en la industria, oficinas, política, universidad, etc., plantean la necesidad de una exploración, orientación y conocimiento del gran campo de trabajo que tiene frente a ella. Este cambio de las actividades tradicionales de la mujer propio de países con una avanzada evolución social, impone una transformación del rol del hombre en cuanto a sus funciones de padre, jefe del hogar y familia. El varón no puede limitar su acción durante el transcurso de la vida familiar a un simple papel de suministrar recursos económicos, debe tomar mayores responsabilidades frente a la formación de la personalidad de sus hijos, frente a los problemas materiales de vivienda, salud 
y vestuario. Para afrontar estas tareas debe recibir preparación adecuada y adquirir conciencia clara de las responsabilidades que al varón le corresponden (ROSSETTI, 1988, p. 146).

En el área de educación física, en la sección sugerencias metodológicas del segundo ciclo de la enseñanza básica, se señala que la actividad física "entre los 10 y 11 años la gimnasia debe diferir progresivamente, según el sexo, en cuanto a intensidad, a su naturaleza y al método, para lo que se hace necesario que la asignatura esté atendida por una persona del mismo sexo de los alumnos" (Los Programas de Estudios de la Educación General Básica, 1969). Es decir, la concepción es que a medida que niños y niñas van creciendo, necesitarán diferenciar la actividad física que desarrollan en estas clases, puesto que su desarrollo motor es diferenciado según su sexo. En esta concepción, el sustento de la diferenciación son la debilidad o fuerza "naturales" adscritas a los sexos.

En el área orientación, la reforma no hace acotaciones especiales en relación a la orientación vocacional y laboral de ambos sexos. En su fundamentación se devela una concepción centrada en el alumno, apareciendo expresada en términos masculinos:

[...] el hombre necesita tomar conciencia de su condición humana, de su singularidad, de sus valores. El hombre necesita adquirir la información más compleja sobre la comunidad en la que vive y la más clara conciencia del compromiso esencial con ella: familia, escuela, trabajo, cambio social. El hombre debe aprender a poner en juego su condición de ser original con su condición de ser comunitario (ROSSETTI, 1988, p. 146).

Las mujeres nunca son mencionadas como sujetos en el lenguaje utilizado en los textos del nuevo currículo ${ }^{7}$. Durante este periodo, la enseñanza media técnico-profesional adquiere la importancia necesaria para dar a las mujeres amplias posibilidades de acceso a ella. Se abren para ambos sexos ramas del conocimiento y del quehacer tecnológico poco exploradas como sastrería y peluquería para los varones; artes gráficas, hotelería, conservería, mecánica liviana y electrónica para las mujeres. La reforma amplía apreciablemente la cobertura del sistema educativo en sus diferentes niveles.

El año 1968, el gobierno firma un convenio con la UNESCO con el propósito de implementar un proyecto experimental que incentive el acceso de las mujeres a las carreras técnicas, que brinde igualdad de oportunidades a ambos sexos, y que permita a las mujeres participar en el desarrollo social y económico del país. Los objetivos del proyecto se orientan a preparar a las mujeres para su incorporación al conocimiento científico y técnico, buscando también conocer los factores que influyen en el escaso interés de las mujeres por estos campos. El proyecto comienza a implementarse en dos establecimientos de educación técnica: la Escuela Industrial n. 6 de varones y la Escuela de Servicios y Técnicas especializadas n. 6 de niñas; estas instituciones registran incrementos importantes en la matrícula de ambos sexos.

Esta reforma, considera a la educación pilar fundamental para el desarrollo de la personalidad masculina y femenina y la posibilidad de cooperación de ambos sexos en la vida social. Las investigaciones realizadas señalan que, desde el parvulario a la universidad, los varones predominan en los establecimientos coeducacionales, tanto fiscales como particulares, aun cuando en algunos establecimientos fiscales los sexos se distribuyen en forma equilibrada. A nivel nacional en los establecimientos fiscales y particulares es mayor el número de niñas que de niños. Sólo en el sector rural el alumnado está compuesto preferentemente por niños (LEYTON, 1970).

Respecto a la relación género y educación, la ENU establece la educación parvularia para ambos sexos como primer nivel educacional obligatorio, con el objeto de facilitar los procesos educativos familiares y una mayor participación de las mujeres en las tareas de producción (ROSSETTI, 1988). En relación a la 
concepción de género en planes y programas de la reforma de la educación del año 1980, se encuentran algunas indicaciones respecto a la concepción del papel social de ambos sexos, ya sea en los objetivos de los programas o en las sugerencias metodológicas para los y las docentes. Hay referencias al papel de ambos sexos en las asignaturas técnico-manuales, de ciencias naturales y en lo relacionado con el consejo de curso.

En el área de expresión plástica de la enseñanza básica y en especial en el programa de técnico manual se sugiere a los docentes "no hacer diferencias entre hombres y mujeres al realizar los trabajos o elegir un material. No presionar a ningún niño a efectuar un trabajo determinado, pero tampoco negarle la posibilidad de hacer aquel que sea de su preferencia sin hacer discriminaciones en cuanto al sexo" (Planes y Programas de la Educación Básica, 1980). Esta sugerencia positiva para el tratamiento de género en la sala de clases, no vuelve aparecer en ninguna otra asignatura ni en ningún otro nivel (ROSSETTI, 1988).

En el programa de Ciencias Naturales, tanto de octavo año básico como del primer ciclo básico de enseñanza media, aparece el tema de la reproducción humana. Al abordar biológicamente los fundamentos de las diferencias entre los sexos, los roles adscritos se consideran naturales y por lo tanto incuestionables. La complementariedad de los sexos queda situada en el plano familiar sin proyectarla al mundo laboral, político y social. La unidad de reproducción humana de enseñanza media es abordada con una orientación netamente valórica. Teniendo como meta deseable, valores y actitudes positivos referentes a las relaciones con personas de sexo complementario, tratamiento temático orientado a formar una familia en forma responsable. Es así como la sexualidad, tanto para mujeres y hombres, sólo se concibe ligada a la procreación, dejándose de lado el control de la natalidad, el placer y la responsabilidad con el propio cuerpo. En términos cualitativos, los planes y programas revisados son iguales para ambos sexos. Desde una perspectiva histórica representan un avance en cuanto a la calidad de la educación proporcionada a las mujeres. Sin embargo, cuando se especifican las diferencias entre unas y otros, se tiende a consolidar los roles tradicionales para hombres y mujeres.

En términos cuantitativos, en la incorporación de la mujer a la educación aparecen serias modificaciones en la participación femenina, la que sigue creciendo durante los años ochenta, especialmente en la enseñanza secundaria.

Si consideramos la evolución de la matrícula de enseñanza básica durante la década 1975-1985, se comprueban en términos de escolarización dos periodos: hasta 1981 hay una escolarización muy alta para ambos sexos. A partir de 1982 se produce un descenso en la escolarización que coincide con la crisis económica. También existe una escolarización levemente superior de los hombres respecto de las mujeres. En cifras absolutas, existen 57.358 mujeres no escolarizadas y sólo 34.192 hombres.

\section{Consideraciones Finales}

La forma de conclusión, podemos inferir que, cuando consideramos el nexo de continuidad en las propuestas de reformas educativas en el contexto chileno durante los últimos cincuenta años, abordamos las tres propuestas de reforma en los gobiernos de Eduardo Frei Montalva (1964-1970), Salvador Allende Gossens (1970-1973) y el régimen militar de Augusto Pinochet Ugarte (1973-1990). Este período histórico, marcado por profundas transformaciones políticas, económicas, sociales y culturales, nos permitió hacer un breve análisis descriptivo en el que se basaban las propuestas y cómo se relacionaban con los temas de género.

La primera conclusión que podemos señalar es que ambas propuestas estaban destinadas a apuntalar la transformación social y educativa chilena, elevándola a estándares internacionales. Eduardo Frei Montalva tuvo como eje estratégico la llamada "Revolución en Libertad", que se expresó a partir de tres ejes temáticos, a saber: expansión de la red educativa, educación dirigida al desarrollo económico e implementación de un 
nuevo modelo pedagógico. Esta reforma cambió los ciclos de la educación básica y la dimensión de valor de la educación.

Salvador Allende Gossens, además de continuar la reforma iniciada por Eduardo Frei Montalva, desarrolló una política de "democratización", que puede ser representada en la Escuela Nacional Unificada, creada durante su gobierno. Allende decidió elevar el nivel educativo de las escuelas y ampliar la cobertura del sistema.

En cuanto a la política educativa en el Régimen Militar de Augusto Pinochet Ugarte, se observó que su propuesta educativa apuntaba a lo que se llamó "modernización del sistema". Basado en los principios cristianos, la educación tradicional ganó fuerza, haciendo que la educación esté sujeta a las leyes del mercado. Los cambios en el currículo se orientan hacia la eficiencia administrativa y económica de la educación.

La segunda conclusión que podemos sacar es sobre el tema de género. Aunque las reformas educativas propusieron "libertad", “democratización” y "modernización”, las tres reformas educativas en Chile de 1965, 1973 y 1981 no lograron incluir, de manera sistemática y equitativa, la figura de la mujer en el espacio educativo. Permaneció como una “espina en el talón de Aquiles", el deseo perentorio de valorización de lo femenino en el espacio educativo, laboral y familiar. Al mencionar los datos actuales, y así concluir nuestra exposición, el sitio web 24 horas.cl, el 7 de marzo de 2019, así presentó la mujer chilena en el mercado laboral: "La brecha de salario por género, sin embargo, sigue siendo un obstáculo importante. En Chile -de acuerdo a cifras recolectadas por la OCDE en 2015- las mujeres percibían casi un 15\% menos de sueldo que los hombres". ¿Existe todavía una idea torpe de la superioridad masculina?

\section{Contribución de los Autores}

Problematización y Conceptuación; Flores RCB, Nogueira EN y Rebufel VA; Metodología; Flores RCB, Nogueira EN y Rebufel VA; Análisis; Flores RCB, Nogueira EN y Rebufel VA; Redacción; Flores RCB, Nogueira EN y Rebufel VA.

\section{Notas}

1. Frei Montalva describió así lo que se entiende por "Revolución en Libertad”: "La Revolución en Libertad es un proceso en marcha que, dentro de la Democracia, respetando la persona y los derechos esenciales de todos los chilenos, está liberando al pueblo mediante la reforma educacional, la reforma agraria, la organización popular y la redistribución de los ingresos, y está impulsando el desarrollo y la independencia económica de Chile por medio de la industrialización, la chilenización del cobre y la guerra a la inflación" (FREI, 1968, p. 8).

2. La política de democratización de Salvador Allende tenía los siguientes ejes: Democratización del aparato judicial y previsiones contra el burocratismo, democratización de la administración territorial, democratización de la seguridad social, democratización del crédito, la democratización del Servicio Nacional de Salud, la formación de organismos colegiados en cada institución de gestión, con la participación de los trabajadores, los beneficiarios y los profesionales y la democratización del Sistema Educacional Chileno. En las propias palabras de Allende se puede ver la intención de construir una educación que refundara la nación y el concepto mismo de ser humano. En su discurso de inauguración del año escolar de 1971, Allende señalaba con claridad que, "[p]ara nosotros, toda la sociedad debe ser una escuela, y la escuela debe ser parte integrante de esa gran escuela que debe ser la sociedad. Pero no la tradicional, introvertida, satisfecha de una enseñanza que puede ser bien impartida, pero que no sobrepasa más allá de sus muros; porque pensamos en la escuela abierta, plenamente integrada a los procesos que inquietan, preocupan e interesan a la comunidad [...] construir la nueva vida y la nueva sociedad requiere 
[...] de un nuevo hombre, una nueva voluntad, una nueva responsabilidad y para ello tenemos que prepararnos" (CASTRO, 2008, p. 121).

3. "La Escuela Nacional Unificada (ENU) fue un proyecto de transformación integral de la educación chilena. Fue impulsado por el gobierno de Salvador Allende, tras un largo debate durante 1971 que involucró a diversos actores como docentes, estudiantes, padres y organizaciones sociales. El balance que se hizo por entonces era que la educación debía ser permanente (desde el nivel preescolar y durante toda la vida), democrática, participativa, pluralista y acorde con las necesidades económicas del país. El documento que se elaboró incluía la creación de amplios mecanismos de participación, la integración de los distintos niveles en un solo sistema y la eliminación de las diferencias entre la enseñanza técnica y humanista. A pesar del empeño puesto por el gobierno, el proyecto generó muchas resistencias al sospecharse que detrás de él existía el propósito de instalar una educación ideologizante de tipo socialista. De esta manera, en 1973 se postergó su implementación, al no obtener el apoyo político de la oposición." Disponible en: http://www.memoriachilena.gob.cl/602/w3-article-93514.html. Acceso en: 24 set. 2019.

4. "Este fenómeno, conocido como municipalización, corresponde a uno de los pilares de la modernización del Estado, según los postulados de von Hayeck y Friedman, inspiradores intelectuales del proyecto capitalista desarrollado por la dictadura chilena (Nef. N. 1999/2000). Sin embargo, pese a que los municipios quedan a cargo de la administración de los establecimientos educacionales, el MINEDUC mantiene su tuición sobre los asuntos curriculares y el control de la asistencia de los estudiantes, asunto fundamental para determinar el subsidio" (OLIVA, 2010, p. 316).

5. "La modernización consiste en la adopción de un modelo de desarrollo basado en el intercambio externo y en una reestructuración de los modos de gestión de la sociedad, orientada a desplazar la acción del Estado a favor de los espacios organizados por el mercado; al tenor de lo cual la autora denuncia las estrategias de penetración del Banco Mundial, según los postulados de la agenda educativa de la Comisión Económica Para América Latina (Cepal)” (OLIVA, 2010, p. 323).

6. La investigación comprende que "el género no es el sexo genital, sino el conjunto de atribuciones simbólicas otorgadas al sexo de las personas" (GEBARA, 2010, p. 4), siendo una construcción sociocultural (RICHTER REIMER, 2000; FIORENZA, 1992), un producto social aprendido, representado, institucionalizado y transmitido de generación en generación (GEBARA apud AMORIM, 2007). Es una categoría analítica que "enfatiza el carácter social de las distinciones construidas a partir de las diferencias biológico-sexuales" (RICHTER REIMER, 2005, p. 26). Para Scott, "el género es un elemento constitutivo de las relaciones sociales basado en las diferencias percibidas entre los [...] sexos, es una forma primaria de dar sentido a las relaciones de poder" (1995, p. 86). Según Schüssler Fiorenza, "el género como práctica discursiva sociopolítico-religiosa produce diferencias no solo de sexo, sino también de raza, clase, preferencia sexual, cultura, religión, edad y nacionalidad” (1992, p. 92).

7. Esto nos lleva a comprender que las Reformas Educativas todavía tenían un aspecto patriarcal preponderante. El término patri-kiriarcal utilizado por Richter Reimer abarca los conceptos de patriarcado y kiriarcado. Según Schüssler Fiorenza, “'[p] atriarcado' significa literalmente el poder del padre sobre sus hijos e hijas y sobre otros miembros del clan o del hogar" (2009, p. 133). "En la antigüedad clásica, la kiriarquía era la regla del señor, amo de esclavos, esposo, caballero de nacimiento libre, culto, dueño de propiedades y élite, para quienes eran hombres sin voz y todas mujeres" (FIORENZA, 2009, p. 136). Sin embargo, "la kiriarquía no es simplemente el dominio de los hombres sobre las mujeres. Es un complejo sistema piramidal de dominación que opera a través de la explotación económica y la subordinación vivida” (FIORENZA, 2009, p. 140). Para Wacker, "desde Aristóteles, patriarcado es un término legal, respectivamente político, que se refiere al dominio concreto de las familias pater sobre su hogar, es decir, no solo sobre su propia familia (esposa, hijo e hijas), pero también sobre sus asalariados y esclavos" (2008, p. 50). Según Richter Reimer, "el judaísmo tiene características patriarcales dentro de su contexto histórico más amplio, pero el patriarcado no era exclusivo de la sociedad y religión judía; Era un sistema vigente en todas las sociedades del mar Mediterráneo, ideológicamente y legalmente apoyado dentro del sistema romano. El patriarcado romano es la dominación y la ocupación geopolítica, la explotación física, sexual y psicológica contra todas las personas, la expansión y construcción sobre la base del trabajo esclavo y la imposición de impuestos y tributos. Este patriarcado es la 
macroestructura, dentro de la cual se organizará la vida, la convivencia y la resistencia a partir de microestructuras como el hogar, la comunidad, la asociación profesional" (RICHTER REIMER apud SOUSA, 2012, p. 29). Aquí buscamos llegar a una antítesis de este patriarcado (JOHNSON apud ARTUSO; PINTO, 2013).

\section{Referencias}

AMORIM, D. D. Violência doméstica contra a mulher: estudo sobre os agressores a partir de uma delegacia especializada de atendimento à mulher, 2007. 157 f. Dissertação (Mestrado em Educação, Cultura e Organizações Sociais) - Fundação Educacional de Divinópolis, Universidade do Estado de Minas Gerais, Divinópolis, 2008.

ARTUSO, V.; PINTO, S. S. P. F. A condição das mulheres nos tempos de Jesus e sua inclusão como participante do Reino sob a perspectiva Joanina. Relegens Thréskeia, Curitiba, v. 2, n. 2, p. 2-9, 2013. https:// doi.org/10.5380/ rt.v2i2.35565

CASINELLI, F. Brecha salarial: Mujeres chilenas reciben un 15\% menos de sueldo que los hombres. 24 Horas, 07 mar. 2019. Disponible en: https://www.24horas.cl/data/brecha-salarial-mujeres-chilenas-reciben-un-15-menos-desueldo-que-los-hombres-2661440. Acceso en: 24 set. 2019.

CASTRO, R. O. Representación y acción legislativa de Salvador Allende. In: VÁSQUEZ, D. (ed.). Salvador Allende: vida política y parlamentaria 1908-1973. Santiago de Chile: Ediciones Biblioteca del Congreso Nacional de Chile, 2008. Disponible en: https://www.bcn.cl/publicaciones/obtienearchivo?id=documentos/10221.1/335/3/Salvador. Allende.pdf. Acceso en: 24 set. 2019.

BIBLIOTECA NACIONAL DE CHILE. Escuela Nacional Unificada (ENU). Memória Chilena, 2018. Disponible en: http://www.memoriachilena.gob.cl/602/w3-article-93514.html. Acceso en: 24 set. 2019.

COX, C. La reforma de la educación chilena: contextos, contenidos, implementación, PREAL n. 8. Colección de Estudios Cieplan, Santiago de Chile, n. 45, p. 5-32, jun. 1997.

FIORENZA, E. S. As origens cristãs a partir da mulher: uma nova hermenêutica. Trad. João Rezende da Costa. São Paulo: Paulinas, 1992.

FIORENZA, E. S. Caminhos de Sabedoria: uma introdução à interpretação bíblica feminista. Trad. Mônica Ottermann. São Bernardo do Campo: Nhanduti, 2009.

FREI, E. Lo que Chile está realizando 1965-1968. Mensaje Presidencial presentado en la Cuenta Pública al Congreso Nacional, Santiago de Chile, mayo 1968.

GEBARA, I. O que é teologia feminista? Brasiliense: São Paulo, 2007.

GEBARA, I. Vulnerabilidade, justiça e feminismos. São Bernardo do Campo: Nhanduti, 2010.

LEYTON, M. La experiencia chilena. La reforma educacional 1965-1970. Santiago: CPEIP, 1970.

MOLINA, S. Un Centro de Investigación que abre Surcos. In: MILOS, P. (ed.). CIDE 40 Años. Santiago de chile: Editorial CIDE, 2004. p. 7-12.

NUÑEZ, I. Reformas educacionales e identidad de los docentes. Chile, 1960-1973. Santiago de Chile: Programa Interdisciplinario de Investigaciones en Educación, 1990. p 145-205. (Serie Histórica n. 3.) 
OLIVA, M. A. Política educativa chilena 1965-2009. ¿Qué oculta esa trama? Revista Brasileira de Educação, Rio de Janeiro, v. 15 n. 44, maio-ago, 2010. https://doi.org/10.1590/S1413-24782010000200008

PARTIDO DEMÓCRATA-CRISTIANO (Chile). Un programa y un gobierno. Santiago de Chile: Del Pacífico, 1967. (Colección Biblioteca Nacional de Chile.) Disponible en: http://www.memoriachilena.gob.cl/602/w3-article-9081. html. Acceso en: 24 set. 2019.

RICHTER REIMER, I. O Belo, as Feras e o Novo Tempo. São Leopoldo/São Paulo: CEBI/Paulus, 2000.

RICHTER REIMER, I. Grava-me como selo sobre o teu coração: teologia bíblica feminista. São Paulo: Paulinas, 2005.

RICHTER REIMER, I. Maria, Jesus e Paulo com as mulheres: textos, interpretações ehistória. São Paulo: Paulus, 2013.

ROSSETTI, J. En la escuela también nos enseñan a ser mujeres. Santiago de Chile: Centro de Investigación y Desarrollo de la Educación, 1987.

ROSSETTI, J. La Educación de las Mujeres en Chile Contemporáneo. Santiago de Chile, Centro de Investigación y Desarrollo de la Educación, 1988.

ROSSETTI. J. Educación: La igualdad aparente entre hombres y mujeres. Santiago de Chile: Centro de Investigación y Desarrollo de la Educación, 1994.

SCOTT, J. W. Gênero: uma categoria útil de análise histórica. Educação e Realidade, Porto Alegre, v. 20, n. 2, p. 71-99, jul.-dez. 1995.

SOUSA, M. C. F. E. O papel da mulher no cristianismo primitivo: uma leitura do Quarto Evangelho. 2012. Dissertação (Mestrado em Teologia) - Escola Superior de Teologia, Faculdades Est, São Leopoldo, 2012.

WACKER, M.-T. Fundamentações históricas, hermenêuticas e metodológicas. In: SCHOTTROFF, L.; SCHROER, S.; WACKER, M.-T. (orgs.). Exegese feminista: resultados de pesquisas bíblicas na perspectiva de mulheres. Trad. Monika Ottermann. São Leopoldo/São Paulo: Sinodal; EST/ASTE, 2008.

\section{Sobre los Autores}

Raquel del Carmen Flores Bernal es doctora en Educación por Pontificia Universidad Católica de Chile (PUC Chile). Magister en Educación por la Universidad Las Condes. Directora de Doctorado en Educación y Jefa del Centro de Investigación en Educación de la Universidad Bernardo O’Higgins.

EMIVALDO SILVA NOGUEIRA es doctor y magister en Ciencias de la Religión y Doctorando en Educación por la Pontifícia Universidade Católica de Goiás (PUC Goiás).

VIVIANA REBUFEL es doctorando en Diseño, Gestión y Evaluación de Políticas Públicas de Bienestar Social Universidad de Valencia. Magister en Gestión y Políticas Públicas - Universidad de Chile. 